\title{
Simulação numérica e design construtal aplicados a placas com enrijecedores com diferentes relações altura/espessura
}

\author{
Numerical simulation and constructal design applied to stiffened plates with different \\ height/thickness ratios
}

\author{
C. M. Nogueira ${ }^{1 *}$; M. L. Cunha ${ }^{1}$; R. R. Amaral ${ }^{2}$; G. S. Troina ${ }^{1}$; J. P. S. Lima ; E. \\ D. Santos ${ }^{1,2} ;$ L. A. Isoldi ${ }^{1,2}$ \\ ${ }^{1}$ Programa de Pós-Graduação em Engenharia Oceânica (PPGEO), Escola de Engenharia (EE), Universidade Federal \\ do Rio Grande (FURG), 96203900, Rio Grande-RS, Brasil \\ ${ }^{2}$ Programa de Pós-Graduação em Modelagem Computacional (PPGMC), Escola de Engenharia (EE), Universidade \\ Federal do Rio Grande (FURG), 96203900, Rio Grande-RS, Brasil \\ ${ }^{3}$ Universidade Federal de Goiás (UFG), 74968755, Goiânia-GO, Brasil
}

*carolmtnogueira@gmail.com

\begin{abstract}
Placas finas de aço com enrijecedores são amplamente utilizadas em estruturas navais, construção civil e aeronáutica. Essas placas têm como característica o seu baixo valor de espessura em relação a sua largura e comprimento. Um parâmetro fundamental na análise dessas estruturas é a sua deflexão quando sujeita a um carregamento. Nessa pesquisa foram analisadas placas retangulares com enrijecedores, sujeitas a uma carga transversal uniformemente distribuída. O cálculo analítico dessas estruturas é complexo e limitado a casos específicos mais simples, sendo assim, necessária a utilização da modelagem computacional para a determinação da distribuição de deflexões. As simulações numéricas foram realizadas através do software ANSYS $®$, que utiliza o Método dos Elementos Finitos (MEF), com emprego do elemento bidimensional SHELL281 de 8 nós com malha regular formada por quadriláteros. As diferentes configurações geométricas analisadas foram determinadas através do uso do Método Design Construtal, que transformou parte da espessura de uma placa de referência (sem enrijecedores) em enrijecedores, dispostos nas direções longitudinal e transversal. Essas diferentes configurações geométricas foram simuladas numericamente, caracterizando uma avaliação pela técnica de Busca Exaustiva, permitindo então a análise da influência dos parâmetros geométricos na deflexão da placa. Os resultados obtidos indicam que o aumento na altura dos enrijecedores transversais é mais relevante do que dos longitudinais, implicando em uma melhoria na resistência à deflexão da placa no que tange os deslocamentos máximos e centrais. Além disso, a geometria otimizada resultou em uma redução de $97,92 \%$ na deflexão máxima e $98,81 \%$ na deflexão central em relação à placa de referência.

Palavras-chave: Deflexão, Enrijecedores, Simulação numérica.
\end{abstract}

Steel thin plates with stiffeners are largely used in naval structures, civil construction and aeronautics. The main characteristic of these plates is the low thickness value in relation to their width and length. The fundamental parameter in the analysis of these structures is their deflection when subjected to a load. In this research, rectangular plates with stiffeners, subjected to a uniformly distributed transverse load, were analyzed. The analytical calculation of these structures is complex and limited to simpler specific cases, so it is necessary to use computational modeling to determine the distribution of deflections. The numerical simulations were performed using the ANSYS ${ }^{\circledR}$ software, which uses the Finite Element Method (MEF), through the 8-node SHELL281 two-dimensional element with regular mesh formed by quadrilaterals. The different geometric configurations were determined using the Constructal Design Method, which transformed part of the thickness of a reference plate (without stiffeners) into stiffeners arranged in the longitudinal and transverse directions. These different geometric configurations were simulated numerically, through an evaluation by the exhaustive search technique, allowing the analysis of the influence of the geometric parameters in the plate's deflection. The results indicate that the increase in the height of the transverse stiffeners is more relevant than the longitudinal ones, implying in an improvement in the plate deflection resistance, in reference to the maximum and central displacements. Furthermore, optimized geometry resulted in a reduction of $97.92 \%$ in the maximum deflection and $98.81 \%$ in the central deflection in relation to the reference plate.

Keywords: Defletion, Stiffeners, Numerical Simulation. 


\section{INTRODUÇÃO}

As placas de aço finas são estruturas vastamente utilizadas na construção naval e oceânica. Segundo Jesus (2013) [1], a construção naval brasileira pode ser entendida como um conjunto formado pelos estaleiros navais de grande e médio porte, sendo os estaleiros encarregados da construção e montagem de embarcações (navios, plataformas, etc.).

De acordo com Szilard (2004) [2] as placas (chapas) são estruturas planas, bidimensionais, apresentando uma de suas dimensões, denominada espessura, muito menor que as demais. Podem ser delimitadas por linhas retas ou curvas. São exemplos de estruturas de placas na indústria naval: os cascos de um navio, o convés e sua superestrutura, além de comporem parte dos maquinários e outros dispositivos.

Segundo Lima (2016) [3] essas chapas apresentam espessura fina e consequentemente baixa resistência à flexão, por exemplo, quando comparadas a vigas de maior altura. Por esse motivo, são aplicadas vigas esbeltas com a finalidade de fornecer maior enrijecimento a estas chapas e, consequentemente, melhorar a resistência à flexão. Essas vigas são, em geral, dispostas na direção longitudinal e transversal das placas.

Estudos sobre a deflexão em placas metálicas vêm sendo desenvolvidos por diversos autores. Banai e Pedatzur (2006) [4], por exemplo, utilizaram o Método dos Elementos Finitos (MEF), baseados no modelo de placa ortotrópica e modelo de grelha, para estimar a deflexão máxima nas placas quando submetidas a carregamento transversal uniforme. Em outro estudo, Liu e Wang (2012) [5] analisaram numericamente os efeitos do reforço com enrijecedor em placas retangulares, descrevendo qual a altura, número e arranjo ideias do enrijecedor que fornecem o melhor efeito de fortalecimento. Na mesma linha de pesquisa, Sapountzakis e Katsikadelis (2000) [6] apresentam a solução para placas reforçadas com vigas, por meio da metodologia que isola as vigas da placa, considerando assim as forças e deformações na interface de união entre os elementos. Essa abordagem permite o cálculo dos esforços de cisalhamento nas regiões de união entre placa e enrijecedor. Nesse caso as soluções das equações diferenciais foram realizadas pelo Método da Equação Análoga (AEM). Silva (2010) [7], por exemplo, por meio do software ANSYS® com elementos finitos do tipo SHELL63 e BEAM44 realizou a análise numérica, com o objetivo de verificar a influência da excentricidade no comportamento de pavimentos com poucas vigas.

No que diz respeito a estudos envolvendo o Design Construtal, diversos autores também propuseram algumas contribuições. Troina (2017) [8], em um estudo mais recente, determinou relações geométricas ótimas que minimizassem o valor da deflexão central em placas enrijecidas, submetidas a esforços transversais uniformemente distribuídos. Para esse fim, o autor utilizou o Método Design Construtal e a técnica de otimização por Busca Exaustiva, além do software ANSYS ${ }^{\circledR}$ com o emprego dos elementos finitos bidimensionais (SHELL93) e tridimensionais (SOLID95), para realizar a solução numérica. Por fim, Cunha et al. (2018) [9] apresentaram uma avaliação geométrica de placas enrijecidas submetidas a carregamento transversal uniformemente distribuído. Com esse objetivo, os autores aliaram Design Construtal e busca exaustiva, visando minimizar a deflexão central da placa.

O presente trabalho tem como finalidade a análise numérica de placas com enrijecedores sujeitas à flexão devido a um carregamento transversal uniformemente distribuído. O processo de análise é baseado na influência dos parâmetros geométricos na deflexão central e máxima da placa devido ao carregamento. Busca-se obter, assim, a melhor configuração geométrica do conjunto placa/enrijecedor que minimize essas deflexões.

\section{MATERIAL E MÉTODOS}

\subsection{Método Design Construtal}

A Teoria Construtal parte do princípio que o mundo não foi formado por acidentes aleatórios, acaso e destino, mas sim pela Lei Construtal a qual é base por trás de toda a diversidade. Essa lei tem como definição: para que um sistema de fluxo de dimensões finitas persista no tempo (viva), sua configuração deve evoluir no tempo de forma que facilite o acesso das correntes do sistema (fluido, energia, tensões...) [10]. 
A aplicação da Lei Construtal é realizada por meio do método Design Construtal, que gera configurações que facilitam o fluxo do sistema. Para isso, são consideradas restrições globais, graus de liberdade a serem variados, e um parâmetro de performance a ser maximizado ou minimizado. Então, aplicando esse método ao problema de placas com enrijecedores, é possível gerar diferentes configurações geométricas que foram simuladas numericamente. Isso permite que seja realizada uma busca da geometria otimizada, ou seja, aquela que minimiza as deflexões.

Neste estudo, para a aplicação do método Design Construtal, foi considerada uma placa sem enrijecedores como referência. Além disso, como o método exige que o volume total se mantenha constante durante o processo, a altura, o comprimento e o volume total da placa de referência permaneceram constantes. Sendo assim, parte do volume da placa de referência (retirado da sua espessura) foi convertido em enrijecedores nas direções longitudinal e transversal. Como a espessura dos enrijecedores $\left(t_{s}\right)$ é constante, é possível variar a altura dos enrijecedores na direção longitudinal $\left(h_{l s}\right)$ e transversal $\left(h_{t s}\right)$, permitindo a geração de diferentes combinações. Dessa forma, foi necessário o emprego do parâmetro $\emptyset$ que é a relação entre o volume de material utilizado para os enrijecedores $\left(V_{S}\right)$ e o volume total da placa de referência $\left(V_{r}\right)$.

Os graus de liberdade do problema analisado são: número de enrijecedores longitudinais $\left(N_{l s}\right)$, número de enrijecedores transversais $\left(N_{t s}\right)$, relação entre a altura $\left(h_{l s}\right)$ e a espessura $\left(t_{s}\right)$ dos enrijecedores na direção longitudinal $\left(h_{l s} / t_{t s}\right)$ e a relação entre a altura $\left(h_{t s}\right)$ e a espessura $\left(t_{s}\right)$ dos enrijecedores na transversal $\left(h_{t s} / t_{s}\right)$.

Para ilustrar o problema, a Figura 1 apresenta um modelo de placa enrijecida e seus parâmetros geométricos, com dois enrijecedores longitudinais e três enrijecedores transversais.

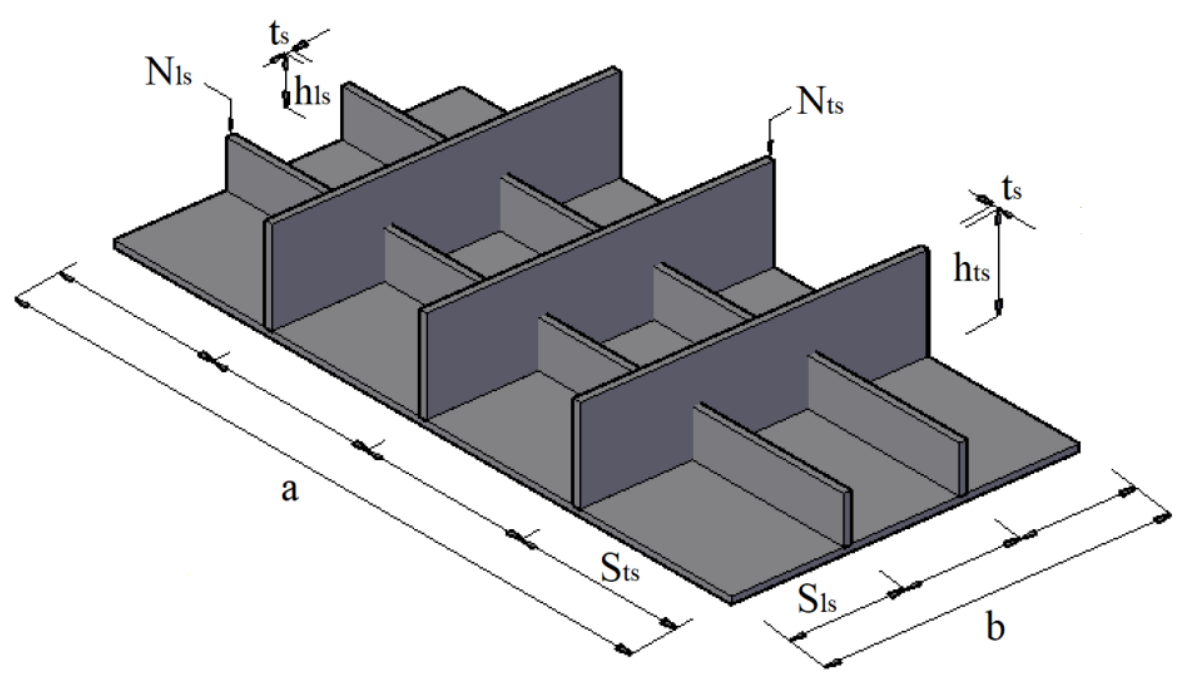

Figura 1: Representação da placa enrijecida na configuração $P(2,3)$.

A fração de volume $\emptyset$ foi definida pelas equações:

$$
\begin{aligned}
& \phi=\frac{V_{s}}{V_{r}}=\frac{N_{l s}\left(a h_{l s} t_{s}\right)+N_{t s}\left(b h_{t s} t_{s}\right)-N_{l s} N_{t s} t_{s}^{2}}{a b t} \quad \text { quando } \quad h_{l s}=h_{t s} \\
& \phi=\frac{V_{s}}{V_{r}}=\frac{N_{l s}\left(a h_{l s} t_{s}\right)+N_{t s}\left(b h_{t s} t_{s}\right)-N_{l s} N_{t s} t_{s}^{2} h_{t s}}{a b t} \quad \text { quando } \quad h_{l s}>h_{t s} \\
& \phi=\frac{V_{s}}{V_{r}}=\frac{N_{l s}\left(a h_{l s} t_{s}\right)+N_{t s}\left(b h_{t s} t_{s}\right)-N_{l s} N_{t s} t_{s}^{2} h_{l s}}{a b t} \quad \text { quando } \quad h_{l s}<h_{t s}
\end{aligned}
$$

onde $V_{s}$ é o volume de material convertido em enrijecedores, $V_{r}$ o volume total de material da placa de referência, $N_{l s}$ e $N_{t s}$ são, respectivamente, o número de enrijecedores nas direções longitudinal e transversal da placa; $a, b$ e $t$ são, respectivamente, o comprimento, a largura e a espessura da 
placa; $h_{l s}$ e $h_{t s}$ são, respectivamente, a altura do enrijecedor na direção longitudinal e transversal e ts é a espessura dos enrijecedores, que é a mesma nas duas direções da placa.

A placa não enrijecida adotada como referência apresenta comprimento $a=2 \mathrm{~m}$, largura $b=$ $1 \mathrm{~m}$, espessura $t=20 \mathrm{~mm}$, seus bordos encontram-se simplesmente apoiados (incluindo as bordas dos enrijecedores) e está submetida a um carregamento transversal uniformemente distribuído de $10 \mathrm{kPa}$. O material de fabricação da placa possui módulo de elasticidade $E=200 G P a$ e coeficiente de Poisson $v=0,3$. No presente estudo foi considerada uma fração de volume $\phi=0,3, N_{l s}=2 \mathrm{e}$ $N_{t s}=3$, e fixou-se a variação do grau de liberdade $h_{t s} / h_{l s}$ entre 0,5 e 2,0 . Os valores considerados para a espessura dos enrijecedores foram: $6,35 \mathrm{~mm}, 12,7 \mathrm{~mm}$ e $25,4 \mathrm{~mm}$.

\subsection{Modelo computacional}

A modelagem computacional procura simular fenômenos físicos por meio de um sistema que envolve engenharia, matemática e ciência da computação. Com essa finalidade, o fenômeno é traduzido por um sistema de equações parciais diferenciais (tradução do problema de engenharia para a matemática), sendo estas equações aproximadas por um método de discretização como o Método de elementos finitos (matemática para matemática aplicada e para a ciência da computação). Por fim os resultados são comparados com o fenômeno físico em estudo (retornando a um problema de engenharia) [11].

Um método de discretização bastante difundido na análise numérica estrutural é o Método dos Elementos Finitos (MEF) [12]. O software ANSYS ${ }^{\circledR}$ 17.2, baseado no MEF, foi utilizado neste trabalho. O elemento finito adotado foi SHELL281, com oito nós, apresentado na Figura 2. Esse elemento é adequado para modelar estruturas de placas finas a moderadamente espessas, apresentando bons resultados nas análises de deformação.

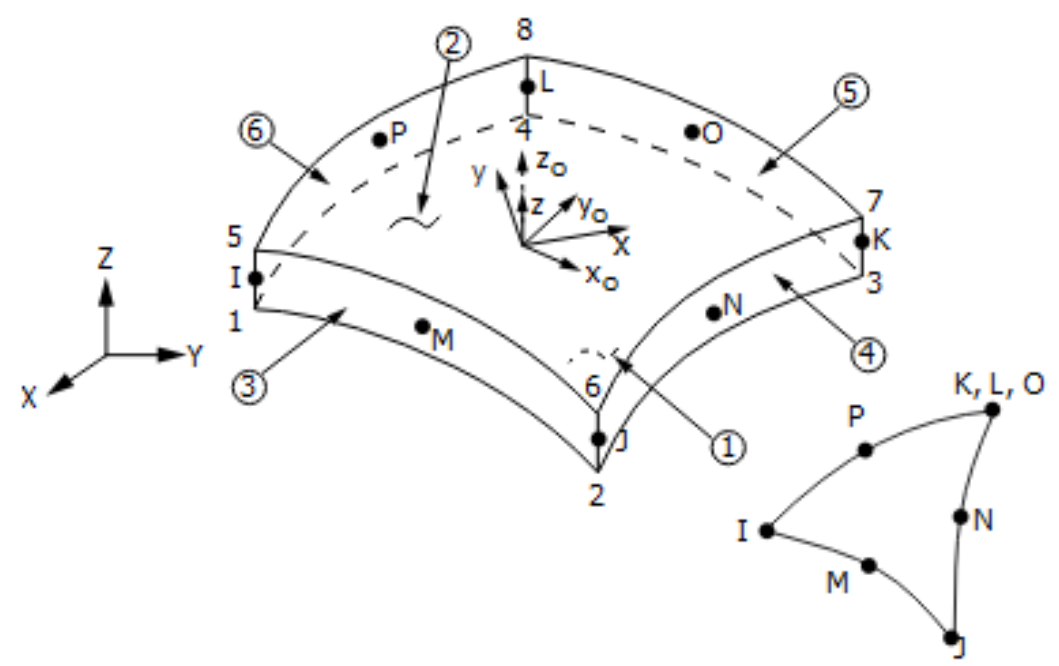

Figura 2: Geometria do elemento, ANSYS SHELL281.

\subsection{Verificação do modelo computacional}

Todos os estudos realizados através da simulação numérica necessitam de verificação e/ou validação do modelo, com o intuito de estimar a precisão do mesmo. Nesta pesquisa realizou-se a verificação através de comparação dos resultados para a deflexão no centro da placa com resultados obtidos por outros autores da literatura que já realizaram estudos nesta área.

A placa em questão apresenta material com módulo de elasticidade equivalente $E=3,9 \times$ $107 \mathrm{kN} / \mathrm{m}^{2}$, coeficiente de Poisson $v=0,3$, espessura constante $t=20 \mathrm{~mm}$, com maior vão de $18 \mathrm{~m}$ e menor vão de $9 \mathrm{~m}$, além de força distribuída $q=10 \mathrm{kN} / \mathrm{m}^{2}$. A placa é simplesmente apoiada em todas as bordas, apresenta o enrijecedor central na direção do maior vão e as alturas de enrijecedores analisadas foram de $0,60 \mathrm{~m}, 1,25 \mathrm{~m}$ e 2,00 m, além do caso altura do enrijecedor igual a zero (placa sem enrijecedor). Desta forma, foi analisada a influência da variação da altura do 
enrijecedor em uma placa retangular na sua deflexão central. Esse problema já foi resolvido numericamente por Sapountzakis e Katsikadelis (2000) [6], Silva (2010) [7] e Troina (2017) [8].

A Tabela 1 apresenta um comparativo entre os resultados numéricos para o deslocamento central da placa, demostrando que os valores convergem com as referências citadas. Pode-se perceber que os resultados apresentados por Sapountzakis e Katsikadelis (2000) [6] apresentam divergência quando comparado com os demais modelos. Segundo Silva (2010) [7], essa diferença ocorre devido à diferença de formulação entre o Método da Equação Análoga (AEM) e o Método dos elementos finitos.

O elemento finito empregado por Troina (2017) [8] é o SOLID95 - Hexaédrico, e segundo o autor, pela sua quantidade de nós e regularidade da malha, tende a obter resultados com maior acurácia, o que justifica a diferença máxima de 6,8\% entre os resultados, salienta-se ainda que os resultados apresentados por Troina (2017) [8] para o elemento SHELL93 - Quadrilátero não obtiveram diferença superior a 1,9\% em relação aos resultados obtidos nesse estudo. Portanto, é possível concluir que o modelo computacional foi satisfatoriamente verificado.

Tabela 1: Verificação do modelo computacional.

\begin{tabular}{ccccc}
\hline & \multicolumn{4}{c}{ Estudos } \\
\cline { 2 - 5 } $\boldsymbol{h}_{\boldsymbol{s}}$ & $\begin{array}{c}\text { Sapountzakis } \mathbf{e} \\
\text { Katsikadelis } \\
(\mathbf{2 0 0 0})(\mathbf{m m})\end{array}$ & $\begin{array}{c}\text { Silva }(\mathbf{2 0 0 0}) \\
\mathbf{( m m})\end{array}$ & $\begin{array}{c}\text { Troina (2017) } \\
(\mathbf{m m})\end{array}$ & $\begin{array}{c}\text { Presente } \\
\text { Estudo } \\
(\mathbf{m m})\end{array}$ \\
\hline $\mathbf{0 , 0 0}$ & 32,00 & 32,20 & 32,76 & 32,87 \\
$\mathbf{0 , 6 0}$ & 4,20 & 16,10 & 15,18 & 16,29 \\
$\mathbf{1 , 2 5}$ & 0,70 & 4,50 & 4,69 & 4,73 \\
$\mathbf{2 , 0 0}$ & 0,20 & 1,60 & 1,67 & 1,66 \\
\hline
\end{tabular}

\subsection{Teste de independência de malha}

O tamanho do elemento finito utilizado na malha necessita ser avaliado de forma que a variação do seu tamanho não altere o resultado, ou seja, deve haver convergência de valores. Portanto, o modelo ideal é aquele se aproxima da realidade do problema físico em um tempo de processamento adequado.

Com esse objetivo, foram realizadas sete simulações numéricas na placa com a maior diferença entre as alturas dos enrijecedores na direção longitudinal e transversal, a placa $\mathrm{P}(2,3) \operatorname{com} t_{s}=$ $6,35 \mathrm{~mm}$ e $h_{t s} / h_{l s}=2$, sendo essa a geometria de maior complexidade geométrica entre as estudadas neste trabalho. A malha definida como independente foi a malha 5 com diferença relativa de $0,25 \%$ para a deflexão central, como é demonstrado na Tabela 2.

Tabela 2: Teste de independência de malha.

\begin{tabular}{cccccc}
\hline Malha & $\begin{array}{c}\text { Tamanho do } \\
\text { elemento }(\mathbf{m m})\end{array}$ & $\begin{array}{c}\left(\boldsymbol{U}_{z}\right)_{\text {max }} \\
(\mathbf{m m})\end{array}$ & $\begin{array}{c}\left(\boldsymbol{U}_{z}\right)_{\text {central }} \\
(\mathbf{m m})\end{array}$ & $\begin{array}{c}\text { Diferença } \\
\text { relativa } \\
\mathbf{m a x} \\
\mathbf{( \% )}\end{array}$ & $\begin{array}{c}\text { Diferença } \\
\text { relativa } \\
\text { centro } \\
(\boldsymbol{\%})\end{array}$ \\
\hline $\mathbf{1}$ & 300 & 0,0152 & 0,00805 & 13,16 & 0,50 \\
$\mathbf{2}$ & 200 & 0,0132 & 0,00801 & 3,79 & 0,62 \\
$\mathbf{3}$ & 100 & 0,0137 & 0,00806 & 1,46 & 0,25 \\
$\mathbf{4}$ & 50,0 & 0,0139 & 0,00804 & 1,44 & 0,25 \\
$\mathbf{5}$ & 25,0 & 0,0141 & 0,00802 & 0,00 & 0,25 \\
$\mathbf{6}$ & 12,5 & 0,0141 & 0,00804 & 0,00 & 0,00 \\
$\mathbf{7}$ & 6,25 & 0,0141 & 0,00804 & 0,00 & 0,00 \\
\hline
\end{tabular}




\section{RESULTADOS E DISCUSSÃO}

A placa não enrijecida utilizada como referência apresentou um deslocamento central (máximo) de $U_{z}=0,69758 \mathrm{~mm}$, o qual foi determinado por simulação numérica. Em relação as placas enrijecidas analisadas, as Figuras 3, 4 e 5 e a Tabela 3 apresentam a variação da deflexão máxima e central da placa, $\left(U_{z}\right)_{\max }$ e $\left(U_{z}\right)_{\text {central }}$, respectivamente, devido à variação do grau de liberdade $h_{t s} / h_{l s}$, tendo como parâmetro a variação da relação nas alturas de enrijecedores da forma, $0,5<$ $h_{t s} / h_{l s}<2,0$ e para três espessuras de enrijecedores $t_{s}=6,35 \mathrm{~mm}, t_{s}=12,70 \mathrm{~mm}$ e $t_{s}=$ $25,40 \mathrm{~mm}$.

Pode-se observar pelos dados apresentados que, como já era esperado, os valores de deflexão da placa para o ponto central e para a deflexão máxima diminuem com o emprego dos enrijecedores, visto que os valores de deflexão de todas as placas analisadas foram menores que os das placas sem enrijecedores, cujo valor de deflexão foi $\left(U_{z}\right)_{\max }=\left(U_{z}\right)_{\text {central }}=0,69758 \mathrm{~mm}$.

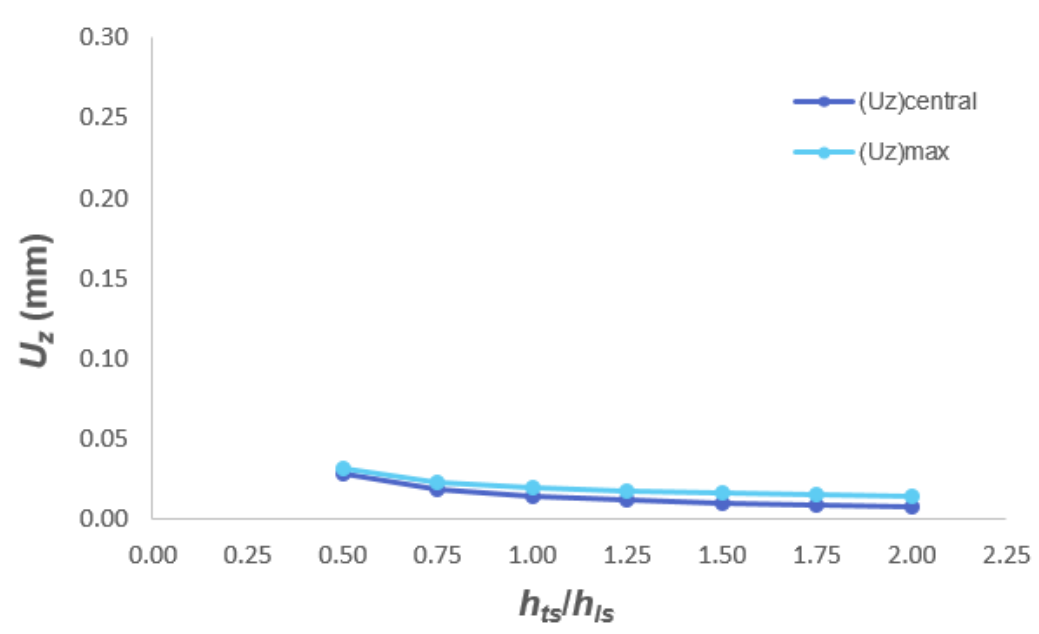

Figura 3: Variação do deslocamento em função da variação do grau de liberdade $h_{t s} / h_{l s}$ para $t_{s}=6,35 \mathrm{~mm}$.

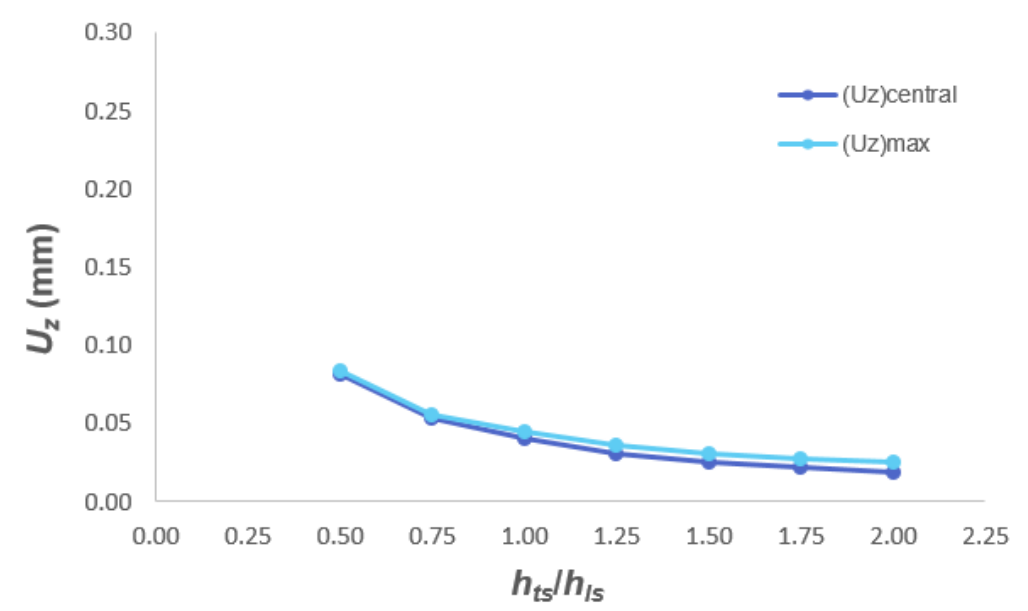

Figura 4: Variação do deslocamento em função da variação do grau de liberdade $h_{t s} / h_{l s}$ para $t_{s}=12,70$ mm. 


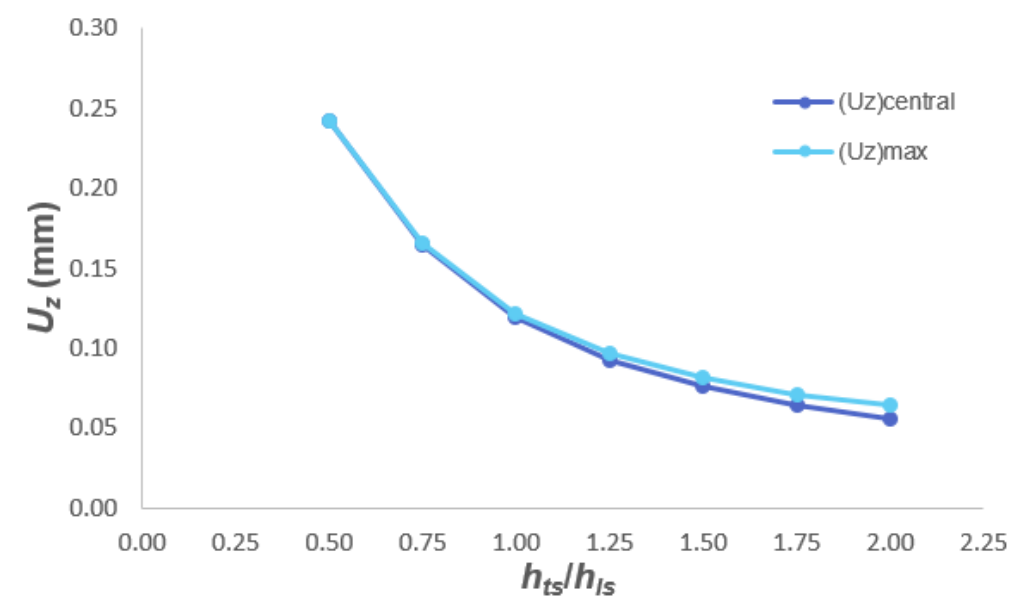

Figura 5: Variação do deslocamento em função da variação do grau de liberdade $h_{t s} h_{l s}$ para $t_{s}=25,40$ mm.

Tabela 3: Valores de deflexão máxima e central conforme variação de $h_{t s} / h_{l s}$ e $t_{s}$

\begin{tabular}{|c|c|c|c|c|c|c|}
\hline \multirow[b]{2}{*}{$t_{s}(\mathbf{m m})$} & \multicolumn{5}{|c|}{ Título } & \multirow[b]{2}{*}{$\begin{array}{c}\text { Diferença } \\
\text { entre } \\
\left(U_{z}\right)_{\max } \mathrm{e} \\
\left(U_{z}\right)_{\text {central }} \\
(\%)\end{array}$} \\
\hline & $\left(\boldsymbol{h}_{t s} / \boldsymbol{h}_{l s}\right)$ & $\boldsymbol{h}_{l s}(\mathbf{m})$ & $\boldsymbol{h}_{t s}(\mathbf{m})$ & $\begin{array}{c}\left(U_{z}\right)_{\max } \\
(\mathbf{m m})\end{array}$ & $\begin{array}{l}\left(\boldsymbol{U}_{z}\right)_{\text {central }} \\
(\mathbf{m m})\end{array}$ & \\
\hline 6,35 & 0,50 & 0,344788 & 0,172394 & 0,031904 & 0,027999 & 12,24 \\
\hline 6,35 & 0,75 & 0,303751 & 0,227813 & 0,022701 & 0,018270 & 19,52 \\
\hline 6,35 & 1,00 & 0,271444 & 0,271444 & 0,019970 & 0,014821 & 25,78 \\
\hline 6,35 & 1,25 & 0,245045 & 0,306306 & 0,017616 & 0,012106 & 31,28 \\
\hline 6,35 & 1,50 & 0,223326 & 0,334989 & 0,016148 & 0,010356 & 35,87 \\
\hline 6,35 & 1,75 & 0,205144 & 0,359002 & 0,015173 & 0,009152 & 39,68 \\
\hline 6,35 & 2,00 & 0,189699 & 0,379398 & 0,014502 & 0,008280 & 42,90 \\
\hline 12,70 & 0,50 & 0,172995 & 0,086498 & 0,083306 & 0,081361 & 2,33 \\
\hline 12,70 & 0,75 & 0,152576 & 0,114432 & 0,056038 & 0,052924 & 5,56 \\
\hline 12,70 & 1,00 & 0,136469 & 0,136469 & 0,044412 & 0,040123 & 9,66 \\
\hline 12,70 & 1,25 & 0,123131 & 0,153914 & 0,036228 & 0,031133 & 14,06 \\
\hline 12,70 & 1,50 & 0,112168 & 0,168252 & 0,031195 & 0,025460 & 18,38 \\
\hline 12,70 & 1,75 & 0,102998 & 0,180246 & 0,027892 & 0,021638 & 22,42 \\
\hline 12,70 & 2,00 & 0,095214 & 0,190427 & 0,025606 & 0,018926 & 26,09 \\
\hline 25,40 & 0,50 & 0,087105 & 0,043553 & 0,242630 & 0,242380 & 0,10 \\
\hline 25,40 & 0,75 & 0,076999 & 0,057749 & 0,166040 & 0,164790 & 0,75 \\
\hline 25,40 & 1,00 & 0,068994 & 0,068994 & 0,121590 & 0,118910 & 2,20 \\
\hline 25,40 & 1,25 & 0,062183 & 0,077729 & 0,096771 & 0,092594 & 4,32 \\
\hline 25,40 & 1,50 & 0,056596 & 0,084894 & 0,081239 & 0,075664 & 6,86 \\
\hline 25,40 & 1,75 & 0,051930 & 0,090878 & 0,070394 & 0,064141 & 8,88 \\
\hline 25,40 & 2,00 & 0,047975 & 0,095950 & 0,063772 & 0,055924 & 12,31 \\
\hline
\end{tabular}

Nota-se, ainda, que com o aumento do valor da relação $h_{t s} / h_{l s}$ há uma tendência de diminuição dos valores, tanto da deflexão máxima quanto no ponto central. Tal fato demonstra que os enrijecedores transversais possuem uma maior influência na redução dos deslocamentos, em comparação com os enrijecedores dispostos longitudinalmente. Em outras palavras, se torna mais vantajoso empregar uma maior parcela de volume de material nos enrijecedores transversais.

Observando-se a Tab. 3 também é possível perceber que o aumento da altura do enrijecedor transversal em relação ao longitudinal acarreta em uma elevação da diferença entre o valor de 
deflexão máxima e central. Essa diferença é maior com a espessura de enrijecedor de 6,35 $\mathrm{mm}$, chegando a $43 \%$ com a relação $h_{t s} / h_{l s}=2$.

Além disso, pode-se observar que a variação do grau de liberdade $h_{t s} / h_{l s}$ apresentou maior impacto nos deslocamentos com o aumento da espessura dos enrijecedores (ts). Para $t_{s}=$ $6,35 \mathrm{~mm}$, observaram-se diferenças de até $55,80 \%$ no deslocamento máximo e $74,35 \%$ no deslocamento central, enquanto a espessura dos enrijecedores de $25,40 \mathrm{~mm}$ apresentou diminuições de até $73,72 \%$ e 76,93\% para o deslocamento máximo e central, respectivamente.

Por fim, os dados apresentados indicam que o aumento na altura do enrijecedor na direção transversal, $h_{t s}$, tem maior impacto na redução da deflexão, principalmente quando é utilizada uma espessura menor de enrijecedor. Teve-se, como melhor configuração estudada, a geometria com $t_{s}$ $=6,35 \mathrm{~mm}$ e $h_{t s} / h_{l s}=2 \mathrm{com}$ uma melhoria de $97,92 \%$ na deflexão máxima e de $98,81 \%$ na deflexão central em relação à placa de referência. Na Figura 6, é possível observar um comparativo entre o campo de deslocamentos da geometria ótima e da placa não enrijecida utilizada como referência. Nota-se que os resultados seguem os princípios do Método Design Construtal, em que ocorre uma ótima distribuição (ou fluxo) de deslocamentos máximos na placa enrijecida, ou seja, melhor distribuição dos deslocamentos máximos na área da placa enrijecida. Tal fato não ocorre na placa sem elementos enrijecedores.

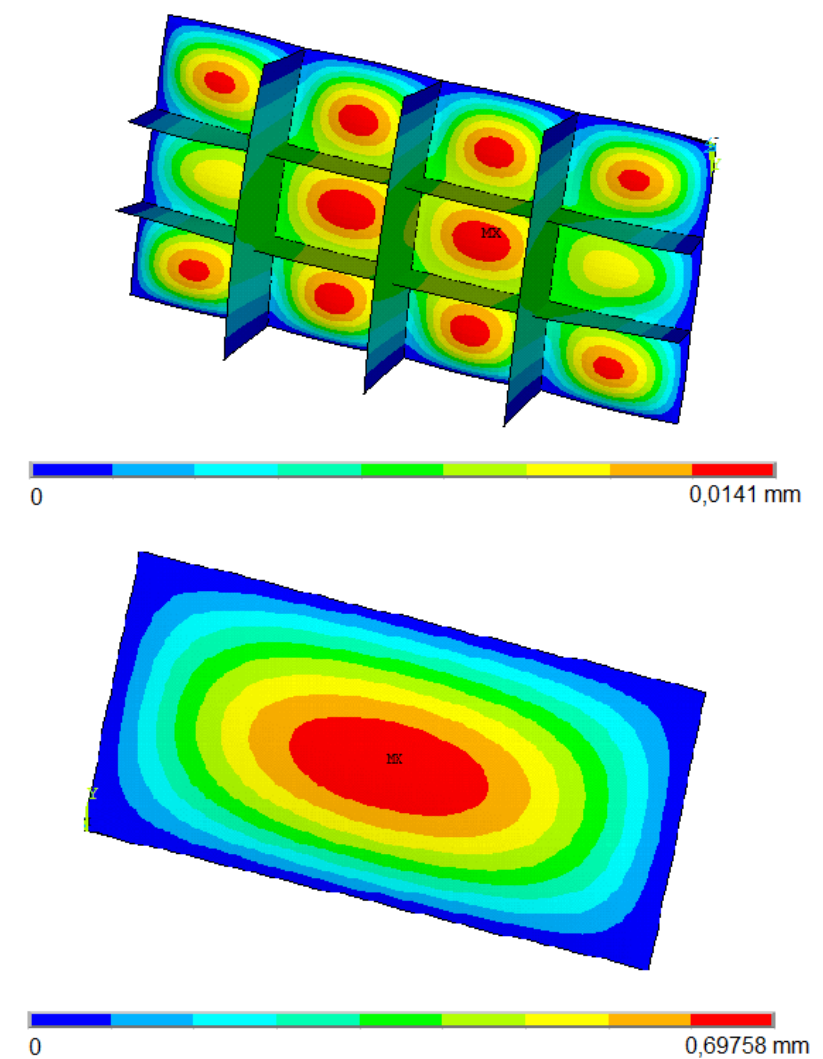

Figura 6: Variação do deslocamento em função da variação do grau de liberdade hts/hls para ts = 6,35 mm.

\section{CONCLUSÃO}

O objetivo do trabalho foi associar a simulação numérica através do software ANSYS $®$, baseado no MEF, com o Método Design Construtal avaliando a influência da configuração geométrica de placas com enrijecedores em relação à deflexão máxima e central quando sujeitas a um carregamento transversal uniformemente distribuído.

Como já observado em diversos estudos na literatura, a adição de enrijecedores, mesmo sem o acréscimo de volume ao conjunto, devido apenas à transformação de volume da placa de referência 
em elementos enrijecidos, apresenta resultados significativos em relação à redução da deflexão de placas metálicas.

Com base nos resultados percebe-se que a consideração de altura de enrijecedor transversal superior à altura do enrijecedor longitudinal teve resultados melhores na diminuição da deflexão da placa quando comparada à placa sem enrijecedores, ambas sujeitas ao mesmo carregamento, isto pode ser explicado pelo fato que o momento fletor solicitante é maior na direção do menor vão [13].

Pôde-se observar, ainda, que o grau de liberdade $h_{t s} / h_{l s}$ tem grande influências nas deflexões da placa enrijecida, uma vez que houve uma redução de $73,72 \%$ e $76,93 \%$ para os deslocamentos máximo e central, respectivamente, placa com $t s=12,70 \mathrm{~mm}$. Demonstrando que um aumento na altura dos enrijecedores transversais, mesmo sem alterar o volume total de material empregado na placa, implica em uma melhoria no comportamento mecânico no que tange os deslocamentos máximos e centrais.

A geometria ótima obtida no presente estudo foi aquela com espessura de enrijecedor $t s=$ $6,35 \mathrm{~mm}$ e $h_{t s} / h_{l s}=2$, cujos deslocamentos máximo e central foram, respectivamente, 0,00802 mm e $0,0141 \mathrm{~mm}$. Então, a geometria otimizada acarretou a uma redução de 97,98 \% na deflexão máxima e de 98,58 \% na deflexão central em relação a placa de referência.

Em trabalhos futuros, pode-se analisar a influência do parâmetro $h_{t s} / h_{l s}$ para outros valores de espessura de enrijecedores. Além disso, é possível acrescentar mais graus de liberdade ao variarse, além dos parâmetros citados, o número de enrijecedores longitudinais $N_{l s}$ e transversais $N_{t s}$.

\section{AGRADECIMENTOS}

Os autores agradecem à FAPERGS, a CAPES e ao CNPq pelo apoio financeiro para o desenvolvimento desta pesquisa.

\section{REFERÊNCIAS BIBLIOGRÁFICAS}

1. Jesus CG. Retomada da indústria de construção naval brasileira: reestruturação e trabalho [Tese]. Campinas (SP): Universidade Estadual de Campinas; 2013. 195 p.

2. Szilard R. Theories and Applications of Plates Analysis. 1 a ed. New Jersey: John Wiley \& Sons; 2004, doi: 10.1002/9780470172872.

3. Lima JP. Análise numérica da flambagem de placas finas de aço com enrijecedores através do método design Construtal [Dissertação]. Rio Grande (RS): Universidade Federal do Rio Grande; 2016. 146 p.

4. Banai L, Pedatzur O. Computer implementation of an orthotropic plate model for quick estimation of the maximum deflection of stiffened plates. Ships Offshore Struc. 2006 Jul; 1(4):323-333, doi:10.1533/saos.2005.0135.

5. Liu Y, Wang Q. Computational study of strengthening effects of stiffeners on regular and arbitrarily stiffened plates. Thin-Walled Struc. 2012 Oct; 59(1):78-86, doi:10.1016/j.tws.2012.05.001.

6. Sapountzakis EJ, Katsikadelis JT. Analysis of Plates Reinforced with Beams Computational Mechanics. Comput Mech. 2000 April;26:66-74, doi:10.1007/s004660000.

7. Silva HBS. Análise numérica da influência da excentricidade na ligação placa-viga em pavimentos usuais de edifícios [Dissertação]. São Paulo (SP): Universidade Federal de São Carlos; 2010. 196 p.

8. Troina GS. Simulação numérica e Design Construtal aplicados à otimização geométrica de placas finas de aço com enrijecedores submetidas a carregamento transversal uniforme [Dissertação]. Rio Grande (RS): Universidade Federal do Rio Grande; 2017. $171 \mathrm{p}$.

9. Cunha ML, Troina GS, Santos ED, Rocha LAO, Isoldi LA. Computational modeling and Constructal Design method applied to the geometric optimization of stiffened steel plates subjected to uniform transverse load. Eng Struc Materials. 2018 Jan; 4(3):139-149, doi:10.17515/resm2017.18st1118.

10. Bejan A, Lorente S. Design with Constructal Theory. Hoboken: Wiley; 2008. 529 p. doi: 10.1002/9780470432709

11. Devloo PRB. Simulação numérica. Multi Ciência: A Linguagem da Ciência. 2005 Mai;4(1):1-13.

12. Azevedo, AFM. Métodos dos elementos Finitos. Porto: Faculdade de Engenharia da Universidade do Porto; 2003. 258 p.

13. Araújo, JM. Curso de concreto armado. 4 ed. Rio Grande: Dunas; 2014. 421 p. 